\title{
Compression of digital holograms of three-dimensional objects using wavelets
}

\author{
Alison E. Shortt, Thomas J. Naughton, and Bahram Javidi* \\ Department of Computer Science, National University of Ireland, Maynooth, \\ County Kildare, Ireland \\ *Electrical and Computer Engineering Department, University of Connecticut, \\ U-2157 Storrs, Connecticut 06269-2157, USA \\ tom.naughton@nuim.ie
}

\begin{abstract}
We present the results of what we believe is the first application of wavelet analysis to the compression of complex-valued digital holograms of three-dimensional real-world objects. We achieve compression through thresholding and quantization of the wavelet coefficients, followed by lossless encoding of the quantized data.
\end{abstract}

(C) 2006 Optical Society of America

OCIS codes: (090.0090) Holography; (100.6890) Three-dimensional image processing; (100.7410 Wavelets); (999.9999) Data compression; (100.2000) Digital image processing.

\section{References and links}

1. J. W. Goodman and R. W. Lawrence, "Digital image formation from electronically detected holograms," Appl. Phys. Lett. 11, 77-79 (1967).

2. M. A. Kronod, N. S. Merzlyakov, and L. P. Yaroslavskii, "Reconstruction of a hologram with a computer," Sov. Phys. Tech. Phys. 17, 333-334 (1972).

3. J. H. Bruning, D. R. Herriott, J. E. Gallagher, D. P. Rosenfeld, A. D. White, and D. J. Brangaccio, "Digital wavefront measuring interferometer for testing optical surfaces and lenses," Appl. Opt. 13, 2693-2703 (1974).

4. L. Onural and P. D. Scott, "Digital decoding of in-line holograms," Opt. Eng. 26, 1124-1132 (1987).

5. U. Schnars and W. P. O. Jüptner, "Direct recording of holograms by a CCD target and numerical reconstruction," Appl. Opt. 33, 179-181 (1994).

6. I. Yamaguchi and T. Zhang, "Phase-shifting digital holography," Opt. Lett. 22, 1268-1270 (1997).

7. E. Cuche, F. Bevilacqua, and C. Depeursinge, "Digital holography for quantitative phase-contrast imaging," Opt. Lett. 24, 291-293 (1999).

8. T. Kreis, Handbook of Holographic Interferometry: Optical and Digital Methods (Wiley-VCH, Berlin, 2004).

9. B. Javidi and E. Tajahuerce, "Three-dimensional object recognition by use of digital holography," Opt. Lett. 25 610-612 (2000).

10. Y. Frauel, E. Tajahuerce, M. -A. Castro, and B. Javidi, "Distortion-tolerant three-dimensional object recognition with digital holography," Appl. Opt. 40, 3887-3893 (2001).

11. T. J. Naughton, Y. Frauel, B. Javidi, and E. Tajahuerce, "Compression of digital holograms for three-dimensional object reconstruction and recognition," Appl. Opt. 41, 4124-4132 (2002).

12. B. Javidi and F. Okano, Three Dimensional Television, Video, and Display Technologies (Springer, Berlin, 2002).

13. O. Matoba, T. J. Naughton, Y. Frauel, N. Bertaux, and B. Javidi, "Real-time three-dimensional object reconstruction by use of a phase-encoded digital hologram," Appl. Opt. 41, 6187-6192 (2002).

14. H. J. Caulfield, ed., Handbook of Optical Holography (Academic Press, New York, 1979).

15. J. W. Goodman, Introduction to Fourier Optics, 3rd ed. (Roberts \& Company, Englewood, Colorado, 2005).

16. M. Burrows and D. J. Wheeler, "A block-sorting lossless data compression algorithm," Tech. Rep. 124, Digital Systems Research Center, Palo Alto, California (1994).

17. W. J. Dallas and A. W. Lohmann, "Phase quantization in holograms," Appl. Opt. 11, 192-194 (1972).

18. T. J. Naughton, J. B. Mc Donald, and B. Javidi, "Efficient compression of Fresnel fields for Internet transmission of three-dimensional images," Appl. Opt. 23, 4758-4764 (2003).

19. T. J. Naughton and B. Javidi, "Compression of encrypted three-dimensional objects using digital holography," Opt. Eng. 43, 2233-2238 (2004).

20. T. Nomura, A. Okazaki, M. Kameda, Y. Morimoto, and B. Javidi, "Image reconstruction from compressed encrypted digital hologram," Opt. Eng. 44, 075801 (2005).

\#67986 - \$15.00 USD

(C) 2006 OSA
Received 13 February 2006; revised 16 March 2006; accepted 20 March 2006 3 April 2006 / Vol. 14, No. 7 / OPTICS EXPRESS 2625 
21. D. Kayser, T. Kreis, and W. Jüptner, "Compression of digital holographic data using its electromagnetic field properties,” Optical Information Systems III, B. Javidi, and D. Psaltis, Eds, Proc. SPIE 5908, 97-105, (2005).

22. I. Daubechies, Ten Lectures on Wavelets (Capital City Press, Vermont, 1992).

23. S. Mallat, "A theory for multiresolution signal decomposition: the wavelet representation," IEEE Trans. Pattern Anal. Mach. Intell. 11, 674-693 (1989).

24. R. DeVore, B. Jawerth, and B. Lucier, "Image compression through wavelet transform coding," IEEE Trans. Inform. Theory 38, 719-746 (1992).

25. L. Onural, "Diffraction from a wavelet point of view," Opt. Lett. 18, 846-848 (1993).

26. Y. Sheng, S. Deschênes, and H. J. Caulfield, "Monochromatic electromagnetic wavelets and the Huygens principle," Appl. Opt. 37, 828-833 (1998).

27. M. Liebling, T. Blu, and M. Unser, "Fresnelets: new multiresolution wavelet bases for digital holography," IEEE Trans. Image Process. 12, 29-43 (2003).

28. M. Liebling, T. Blu, and M. Unser, "Complex-wave retrieval from a single off-axis hologram,” J. Opt. Soc. Am. A 21, 367-377 (2004).

29. L. Zeng, C. P. Jansen, S. Marsch, M. Unser, and P. R. Hunziker, "Wavelet compression of four-dimensional arbitrarily size echocardiographic data," IEEE Trans. Med. Imaging 21, 1179-1187 (2002).

30. Z. Zeng and I. G. Cumming, "SAR image data compression using a tree-structured wavelet transform," IEEE Trans. Geoscience and Remote Sensing 39, 546-552 (2001).

\section{Introduction}

Holography is an established technique for recording and reconstructing real-world threedimensional (3D) objects. Digital holography $[1,2,3,4,5,6,7,8]$ has recently become feasible due to advances in megapixel CCD sensors with high spatial resolution and dynamic range. A technique known as phase-shift interferometry $[3,6]$ was used to create our in-line digital holograms $[9,10]$, which are in an appropriate form for processing and transmission. It has been proposed [11] to stream digital holograms over a network to generate a form of 3D video [12]. To reconstruct a particular view of the $3 \mathrm{D}$ object $[14,15]$, an appropriate region of pixels is extracted from the hologram and numerical propagation $[4,6,9]$ is applied. A real-time reconstruction technique using inexpensive components has also been demonstrated [13]. Their large storage requirements mean that to facilitate efficient storage and transmission appropriate compression techniques must be developed.

Lossless image compression techniques, such as Burrows-Wheeler [16] (BW), perform poorly when applied to holograms due to the inherent speckle content that gives the holograms a white-noise appearance [11]. Holographic speckle is difficult to remove directly as it actually contains 3D information. Therefore, lossy compression seems essential. Phase quantization has been applied to holographic data in the past [17]. We extend previous research on quantization compression of holographic data $[11,18,19,20,21]$ by transforming our data to the wavelet domain prior to quantization, and further apply lossless compression to the quantized coefficients. Wavelets have already been used to describe Fresnel propagation of (for example) digital holograms $[25,26,27,28]$.

\section{Digital hologram compression}

Five 3D objects were used in our experiments. Their digital holograms contain $2028 \times 2044$ pixels, with each pixel composed of an 8 byte real value and an 8 byte imaginary value. For practical reasons, we analyzed a single $1024 \times 1024$ pixel window of each hologram. Uniform quantization is defined for an individual pixel $H(x, y)$ as

$$
H^{\prime}(x, y)=\operatorname{round}\left\{H(x, y) \times \sigma^{-1} \times\left[2^{(b-1)}-1\right]\right\}
$$

and is applied to each $x \in\left[1, N_{x}\right], y \in\left[1, N_{y}\right]$, where

$$
\sigma=\max \{|\min [\operatorname{Im}(H)]|,|\max [\operatorname{Im}(H)]|,|\min [\operatorname{Re}(H)]|,|\max [\operatorname{Re}(H)]|\} .
$$

\#67986 - \$15.00 USD

(C) 2006 OSA
Received 13 February 2006; revised 16 March 2006; accepted 20 March 2006 3 April 2006 / Vol. 14, No. 7 / OPTICS EXPRESS 2626 
Here, $N_{x}$ and $N_{y}$ are the number of samples in the $x$ and $y$ directions, respectively, $b$ represents the number of bits per real and imaginary value, $\max (\cdot)$ returns the maximum scalar in its argument(s), and round $(\alpha)$ is defined as $\lfloor\alpha+0.5\rfloor$. All real and imaginary values will then be integers in the range $\left[-2^{(b-1)}+1,2^{(b-1)}-1\right]$. After decompression and prior to object reconstruction, each value is rescaled, by dividing by $2^{(b-1)}-1$, to the $[-1,1]$ interval.

In our compression experiments, a digital hologram $H$ is compressed and then decompressed as $H^{\prime}$, and an object $U^{\prime}$ reconstructed by numerical propagation. The quality of the compressed reconstruction is measured using normalized rms (NRMS) difference, calculated from

$$
D=\left[\sum_{m=0}^{N_{x}-1} \sum_{n=0}^{N_{y}-1}\left\{|U(m, n)|-\left|U^{\prime}(m, n)\right|\right\}^{2} \times\left(\sum_{m=0}^{N_{x}-1} \sum_{n=0}^{N_{y}-1}|U(m, n)|^{2}\right)^{-1}\right]^{1 / 2},
$$

where $(m, n)$ are discrete spatial coordinates in the reconstruction plane. In order to reduce the effects of speckle noise, only amplitude in the reconstruction plane is considered and a $5 \times 5$ pixel mean filtering operation is applied prior to calculation of NRMS difference.

\section{Wavelets}

The continuous wavelet transform of a function $f(x)$ is defined as

$$
g(t, d)=\int f(x) \psi_{t, d}^{*}(x) \mathrm{d} x,
$$

where $*$ denotes complex conjugation and the variables $t$ and $d$ denote translation and dilation factors, respectively. The basis functions, or wavelets, $\psi_{t, d}(x)$ are generated from the mother wavelet $\psi(x)$, a non-zero function in some small interval that explores, through a convolution as shown, the properties of the input data in that interval. A scaling function is used to generate dilated and translated versions of the mother wavelet, as in

$$
\psi_{t, d}(x)=d^{-0.5} \psi[(x-t) / d],
$$

where $d^{-0.5}$ ensures energy normalization across different dilations.

The discrete wavelet transform [22] (DWT) has been applied in fields such as signal processing [23] and image compression [24], and has been found to efficiently represent data exhibiting sharp changes or discontinuities. The basic process involves applying averaging and differencing operations to the input data and retaining the approximation and detail coefficients, respectively, at each resolution level (a step in the DWT). The simplest wavelet function, Haar, calculates (in our case) its complex-valued approximations for resolution level $r$ as $a_{i}^{r}=\left[a_{2 i}^{r-1}+a_{2 i+1}^{r-1}\right] / \sqrt{2}$ and details as $b_{i}^{r}=\left[a_{2 i}^{r-1}-a_{2 i+1}^{r-1}\right] / \sqrt{2}$, where $i$ is a pixel index.

\section{Wavelets applied to digital holograms}

Wavelets have been adapted for the compression of multidimensional data [29] and SAR image data [30], which has similar properties to our data. We modified Matlab's wavelet toolbox in order to apply the DWT to our complex-valued data. Also, since the neighboring pixels in our holograms exhibit no correlation, we chose to employ the 1D DWT. We used 53 discrete mother wavelets, with 1, 3, 10 and 20 resolution levels. Figure 1 shows dramatically changing values in the approximation and detail coefficients for 1-20 resolution levels with the Haar wavelet in holograms of two 3D objects (a die and a bolt). If all coefficients are considered together for clustering, then resolution levels with a narrow range of coefficient values would be quantized with a smaller number of clusters compared to levels with a broader range of coefficient values. Hence there is a need to quantize each level independently in any compression algorithm.

\#67986 - \$15.00 USD

(C) 2006 OSA
Received 13 February 2006; revised 16 March 2006; accepted 20 March 2006 3 April 2006 / Vol. 14, No. 7 / OPTICS EXPRESS 2627 


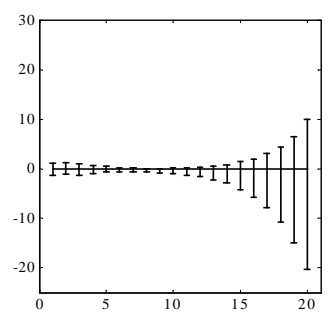

(a)

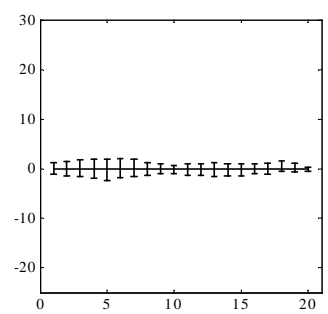

(b)

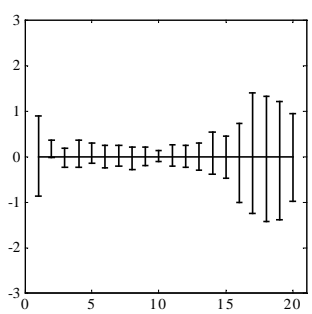

(c)

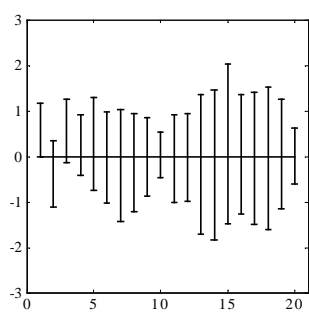

(d)

Fig. 1. Vertical bars illustrating minimum and maximum values at each level of approximation for 20 resolution levels using the Haar wavelet for (a) die and (b) bolt, and each level of detail for (c) die and (d) bolt. $\mathrm{x}$-axis: resolution level, $\mathrm{y}$-axis: value at each level.

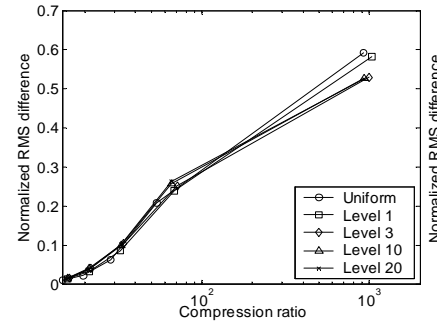

(a)

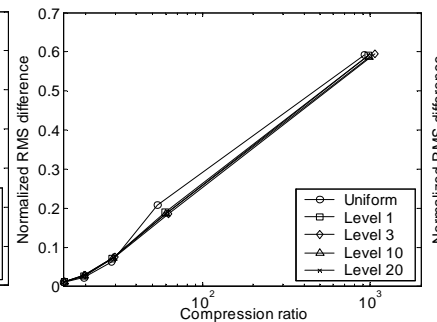

(b)

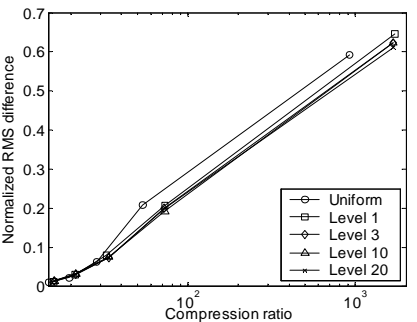

(c)

Fig. 2. NRMS error of the reconstructed object plotted against compression ratio for the die object for wavelet levels 1, 3, 10 and 20 for (a) bior3.7, (b) Haar, and (c) db4.

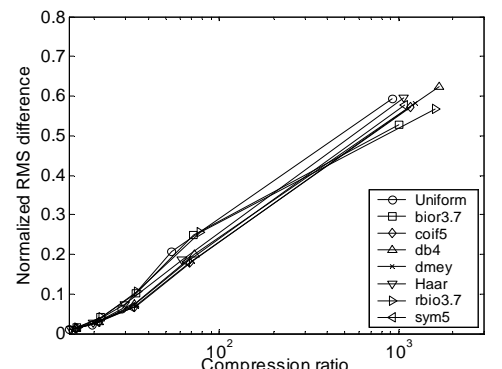

(a)

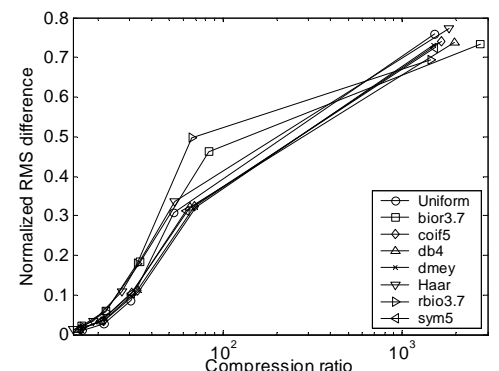

(b)

Fig. 3. NRMS error of the reconstructed object plotted against compression ratio for (a) die, and (b) bolt, for uniform quantization and 7 mother wavelets with 3 resolution levels.

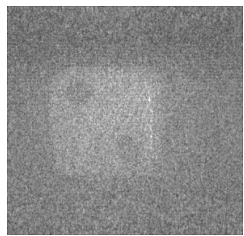

(a)

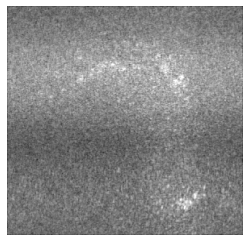

(d)

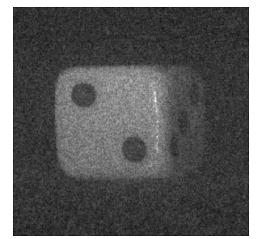

(b)

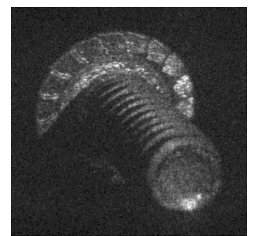

(e)

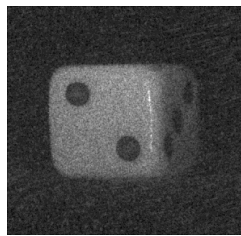

(c)

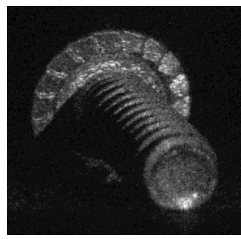

(f)

Fig. 4. Reconstructions of the die and bolt objects, respectively, with (a),(d) 2 bits, (b),(e) 3 bits, and (c),(f) 4 bits uniform quantization of the Haar wavelet coefficients.

\#67986 - \$15.00 USD

(C) 2006 OSA
Received 13 February 2006; revised 16 March 2006; accepted 20 March 2006 3 April 2006 / Vol. 14, No. 7 / OPTICS EXPRESS 2628 


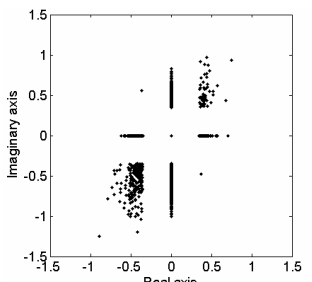

(a - before)

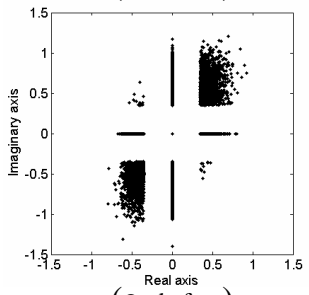

(c - before)

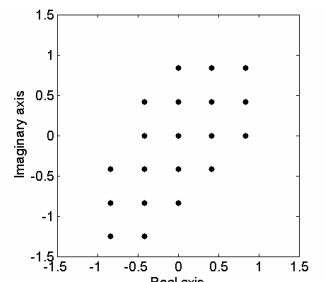

$(\mathrm{a}-$ after $)$

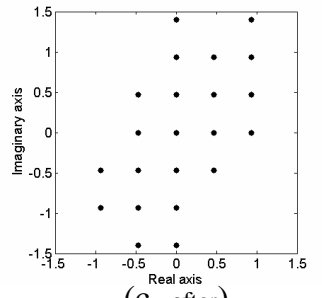

(c - after)

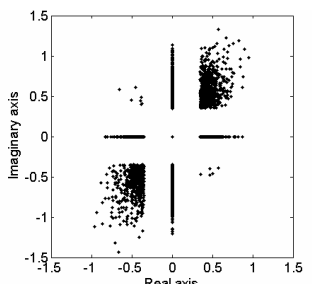

(b - before)

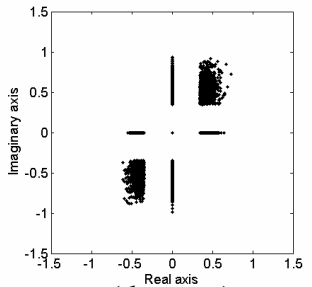

(d - before)

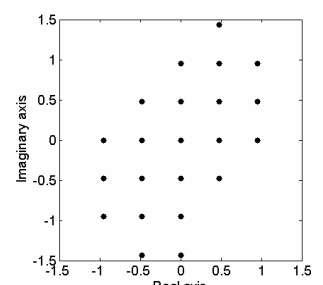

(b - after)

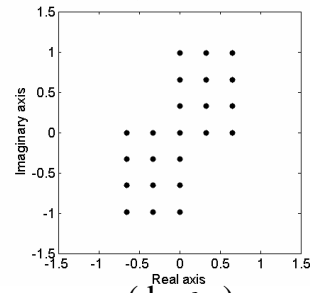

(d $\mathrm{deal}$ - after $)$

Fig. 5. Scatter plots of the complex-valued Haar wavelet coefficients for the die object. Each point in each complex plane represents the value of a thresholded wavelet coefficient. Plots show the distribution of values before and after quantization: (a) approximation, (b) level 3 details, (c) level 2 details, and (d) level 1 details, for a complex threshold of $\pm 0.35 \pm 0.35 i$.

Our approach was to uniformly quantize the wavelet coefficients, apply BW to the concatenation of the real and imaginary streams (previously found to be the best lossless approach for our data [11]), measure the compression ratio, apply the inverse DWT, reconstruct the on-axis view of the 3D object, and calculate NRMS error. The performance of each mother wavelet appeared to depend on the number of resolution levels, the number of quantization bits, and on the hologram. Of the 53 wavelets initially evaluated, we chose a representative set of 7 (bior3.7, coif5, db4, dmey, Haar, rbio3.7 and sym5) for systematic analysis. For clarity, only selected results are shown. Figure 2 shows NRMS error plotted against compression ratio for the die hologram. In a comparison of these 7 wavelet functions, resolution level 3 was deemed to have performed best (lowest NRMS error with the highest compression ratio for a particular number of bits of quantization) over all 5 holograms. Figure 3 shows the results at this resolution level for two holograms. The improved performance of the wavelet functions over uniform quantization in the hologram domain is apparent. Figure 4 shows reconstructions of a $1024 \times 1024$ pixel window of two holograms with quantized Haar wavelet coefficients, with 3 resolution levels.

\section{Combined thresholding and quantization}

We introduced a thresholding technique to improve performance, which sets to zero all complex wavelet coefficients that have a real or imaginary value closer to zero than our threshold, prior to quantization (see Fig. 5). Resolution levels 1, 3, 10 and 20, and threshold values in the range $[0.05+0.05 \mathrm{i}: 1+\mathrm{i}]$ with increments of 0.05 in either dimension, were evaluated. Over the aforementioned seven mother wavelets, and over all five digital holograms, and for quantization of $2-6$ bits, 3 resolution levels was found again to achieve on average the best performance. Figure 6 shows the compression performance at 3 resolution levels, at 2,3, and 4 bits quantization, and for several different thresholds. These plots show that the amount of quantization largely dictates the compression ratio (the distinct clusters for each number of bits) and the different threshold levels perform more of a fine tuning task. The leftmost point on each curve is due to quantization alone (threshold of $\pm 0 \pm 0 \mathrm{i}$ ), and each additional point on the curve represents increased thresholding. The slope of each curve defines the effect of thresholding. It can be seen

\#67986 - \$15.00 USD

(C) 2006 OSA
Received 13 February 2006; revised 16 March 2006; accepted 20 March 2006 3 April 2006 / Vol. 14, No. 7 / OPTICS EXPRESS 2629 


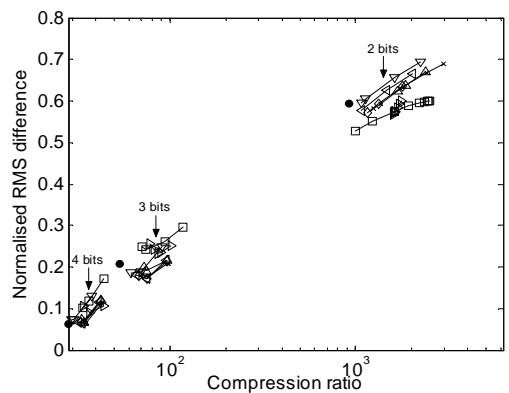

(a)

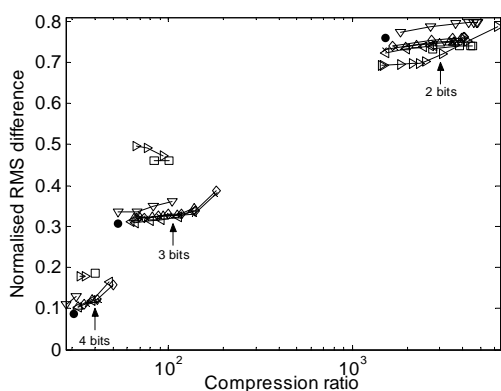

(b)

Fig. 6. NRMS difference of the reconstructed object plotted against compression ratio for hologram (a) die, and (b) bolt, for thresholding and 4, 3 and 2 bit uniform quantization applied directly to the hologram data and seven mother wavelets all with 3 resolution levels. $\bullet$ uniform., $\square$ bior3.7., $\diamond$ coif5., $\triangle$ db4., x dmey., $\nabla$ Haar., $\triangleright$ rbio3.7., $\triangleleft$ sym5.

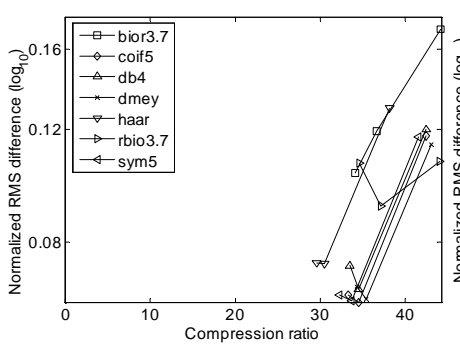

(a)

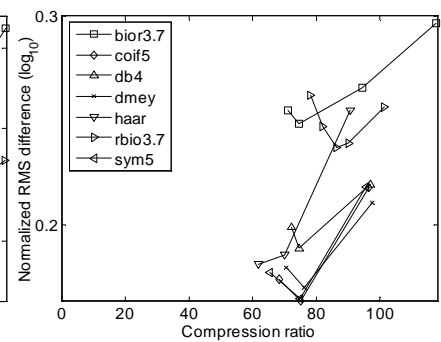

(b)

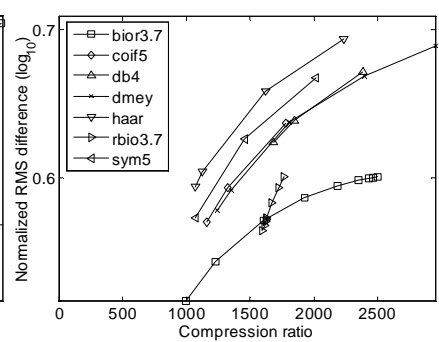

(c)

Fig. 7. NRMS difference of the reconstructed object plotted against compression ratio for die hologram for thresholding and (a) 4 bits, (b) 3 bits, and (c) 2 bits uniform quantization applied directly to hologram data and seven mother wavelets all with 3 resolution levels.

that thresholding achieves improvements in compression ratio for little or no increase in NRMS error. The three clusters in Fig. 6(a) were replotted on separate scales for Fig. 7. We note the surprising observation that most mother wavelets perform comparably for each hologram, and that no single mother wavelet consistently outperforms the others for all quantizations.

\section{Conclusion}

We investigated the use of the DWT for the compression of digital holograms of 3D objects. Of 53 wavelet functions initially tested, we chose a representative sample of seven. A range of resolution levels were examined and it was found that 3 levels performed best on average with our holographic data. The coefficients at each level of detail as well as the approximation coefficients were rescaled independently, due to the diverse range of values we observed in each level, and we applied uniform quantization to the rescaled wavelet coefficients prior to lossless encoding. Thresholding was shown to have the ability to increase compression ratios with little increase in reconstruction error. Although it was expected that several factors would affect compression performance (choice of mother wavelet, choice of hologram, number of resolution levels, number of bits of quantization), it was surprising that no one wavelet function outperformed all others in the study. Although in terms of compression ratio alone, wavelet quantization and uniform quantization are comparable, an analysis of the combined compression ratio and NRMS error shows wavelet quantization to be superior.

The authors wish to thank Enrique Tajahuerce and Yann Frauel for use of their hologram data. This research received the support of Enterprise Ireland and Science Foundation Ireland.

\#67986 - \$15.00 USD

(C) 2006 OSA
Received 13 February 2006; revised 16 March 2006; accepted 20 March 2006 3 April 2006 / Vol. 14, No. 7 / OPTICS EXPRESS 2630 